\title{
Quimioterapia complementaria tras resección de metástasis hepáticas en el carcinoma colorrectal
}

\author{
J. M Viéitez, E. Uña, B. Mareque
}

Se ha calculado que el $60 \%$ de los pacientes intervenidos de un carcinoma colorrectal va a desarrollar metástasis hepática de forma meta crónica y que entre el 15-25\% de los pacientes son diagnosticados con metástasis hepáticas sincrónicas. Además, los resultados de estudios sobre biopsias confirman que las metástasis se mantienen confinadas en el hígado en 1/3 de los pacientes ${ }^{1}$. En base a estos datos se ha intentado mejorar los resultados del carcinoma de colon empleando tratamientos locorregionales sobre el hígado, tanto en enfermedad hepática irresecable como en tratamientos complementarios.

Aunque las metástasis hepáticas se nutren por la arteria hepática, las micrometástasis lo hacen inicialmente a través del flujo venoso de la porta. Esto hizo que fuera la vía portal la que se empleó con más asiduidad para los primeros tratamientos locorregionales. Este es un procedimiento de cierta complejidad técnica y se ensayó tras resección de tumores primarios en un buen número de ensayos ${ }^{2-8}$. Aunque en alguno de ellos se detectó un aumento en la supervivencia, en el mayor de ellos ${ }^{7}$ fue sin mejorar el control hepático, por lo que se atribuyó su efecto al paso sistémico de la droga. Además, el metaanálisis de 1997 demostró un beneficio absoluto en la supervivencia de tan sólo un $4,7 \% \%^{9}$ ), por lo que esta modalidad de tratamiento cayó en desuso (Tabla I).

En cuanto a los tratamientos paliativos intraarteriales, hay una controversia no zanjada. Parece claro que existe un mejor porcentaje de respuestas, e incluso un tiempo más prolongado hasta la progresión; no obstante; tan sólo el metaanálisis publicado en $1996^{10}$ y el estudio de Rougier y col ${ }^{11}$, han podido demostrar una mejoría de la supervivencia. Probablemente esto haya sido porque en algunos estudios se permitió cruzar los brazos ${ }^{12}, 13$, se permitió incluir pacientes con ganglios en hilio hepático ${ }^{13}$ (lo cual amortigua el posible

Servicio de Oncología Médica

Hospital Central de Asturias

Oviedo (Asturias) beneficio al tener enfermedad extrahepática), el número de pacientes de algunos estudios fue muy pequeño ${ }^{14}$, muchos pacientes no recibieron el tratamiento previsto $^{15}$, y en algunos estudios el tratamiento no se comenzó hasta la aparición de síntomas ${ }^{16}$ (se sabe que esto repercute negativamente en la supervivencia) ${ }^{17}$.

Caso contrario ha sido el de los tratamientos intraarteriales complementarios. Conceptualmente no parece tan claro su hipotético beneficio, ya que las micrometástasis se nutren de ramas de la arteria porta. No obstante, es posible que en el contexto de una enfermedad diseminada en el hígado, lo que haya que combatir sea algo mayor que micrometástasis. Desde hace más de 10 años se vienen realizando intentos terapéuticos no aleatorizados en este grupo de pacientes. Los reasultados fueron realmente alentadores, ya que encontraron tasas de supervivencia muy altas ${ }^{18-23}$, controles hepáticos que llegaron al $100 \% 20$, y algunos de ellos observaron diferencias significativas con respecto a controles no aleatoriza$\operatorname{dos}^{18,21,22}$. La variedad de esquemas empleados fue muy grande: $5 \mathrm{Fu}$ intra-arterial directo ${ }^{18}$, fluoropirimidinas en asociación con mitomicina $C^{20,22,23}$, o con interleukina- ${ }^{20}$. Tabla II.

Con respecto a los estudios aleatorizados hay que destacar el de Kemeny $\mathrm{N}_{\text {y }} \mathrm{col}^{24}$, en donde se obtuvieron mejores supervivencias a 2 años, mejores medianas de supervivencias, mejor control hepático y mejor supervivencia libre de progresión, en los pacientes tratados con quimioterapia intraarterial y endovenosa que con quimioterapia endovenosa exclusivamente. Este estudio fue diseñado para aflorar diferencias en la mediana de supervivencia, por lo que su dimensión fue demasiado pequeña como para observar otras diferencias. No obstante ha sido el primer estudio donde se ha podido comprobar algún impacto claro en la evolución de estos pacientes. La mejoría en el control hepático fue evidente, lo que permitió que con el tiempo afloraran lesiones en otros órganos que presumiblemente no se hubieran evidenciado clínicamente de otro modo. Desafortunadamente, clínicamente es muy difícil que los pacientes no "exijan" algún tipo de tratamiento tras una resección de metástasis hepática, por lo que todavía no tenemos el dato de si la QT endovenosa convencional aporta algún beneficio a la cirugía sin tratamiento complementario. Sólo se ha podido constatar que la QT intraarterial junto con la quimioterapia IV prolon- 
TABLA I

Quimioterapia intraportal tras resección de cáncer de colon

\begin{tabular}{|c|c|c|c|c|c|c|}
\hline Autor & Tumor & Estadio & Tto & № pacientes & $\begin{array}{c}\text { de mis } \\
\text { hepáticas }\end{array}$ & $\begin{array}{l}\text { _de } \\
\text { supervivencia }\end{array}$ \\
\hline Taylor (2) & Colon/recto & $A, B, C$ & 5Fu IA vs control & $\begin{array}{l}127 \\
117\end{array}$ & $\mathrm{SI}$ & $\mathrm{SI}$ \\
\hline $\begin{array}{l}\text { Australia- } \\
\text { new Zealand (3) }\end{array}$ & & $B, C$ & 5Fu IP e IV vs control & 372 en total & - & $\mathrm{SI}$ \\
\hline Wereldsma (4) & Colon/Recto & $A, B, C$ & $5 F u \dot{i}^{2}$ vs uroquinasa vs control & $\begin{array}{l}99 \\
103 \\
102\end{array}$ & $\mathrm{SI}$ & NO \\
\hline SAKK (5) & Colon/recto & $A, B, C$ & 5Fu mitomicina $\dot{e}^{2}$ vs control & $\begin{array}{l}252 \\
253\end{array}$ & NO & $\mathrm{SI}$ \\
\hline NCCTG (6) & Colon/recto & $\mathrm{B} 2, \mathrm{C}$ & 5Fu vs control & $\begin{array}{l}110 \\
109\end{array}$ & NO & NO \\
\hline NSABP CO2 (7) & Colon & $A, B, C$ & $5 F u$ vs control & 442 & NO & $\mathrm{SI}$ \\
\hline Fielding (8) & Colon/recto & $A, B, C$ & 5Fu vs Heparina vs control & $\begin{array}{l}130 \\
123 \\
145\end{array}$ & NO & NO \\
\hline
\end{tabular}

IV, intravenoso; IP, intraportal; 5Fu, 5-Fluorouracilo

TABLA III

\section{Resultados de estudios no aleatorizados con quimioterapia intra-arterial}

\begin{tabular}{|c|c|c|c|c|}
\hline Autor & № enf & Tto & Meiana Sup & Control Hepático \\
\hline Curleyy col (18) & 20 & 5Fu IA Dir & 33 & $83 \%$ \\
\hline Lorenz M y col (19) & 60 & 5Fu ó FUdr & 52 & $50 \% *$ \\
\hline Okuno y col (20) & 18 & $\mathrm{IL}-2+5 \mathrm{Fu}+\mathrm{MMC}$ & $28.5+$ & $100 \%$ \\
\hline Ambiru y col (21) & 78 & 5Fu+aclarubicina & 148 & $71 \%$ \\
\hline Kokudo y col (22) & 38 & $5 \mathrm{Fu}+\mathrm{FC}$ ó $M M C+5 \mathrm{Fu}$ & 43 & - \\
\hline Nonami y col (23) & & MMC & $60+$ & - \\
\hline
\end{tabular}

*a 63 meses

ga el intervalo libre de progresión (estudio de Kemeny $M$ y col. en el que el reclutamiento fue extremadamente corto, probablemente debido a que los pacientes no aceptaban el brazo control: 70 pacientes en 7 años $)^{25}$. Existen dos estudios (con resultados positivos) con QT intrarterial y sin QT endovenosa, pero el número de pacientes es tan pequeño que sería aventurado esbozar algún tipo de conclusión ${ }^{26,27}$.

No obstante, no todos los ensayos realizados con QT intraarterial complementaria resultaron positivos, ya que no sirve cualquier fármaco o cualquier esquema para el tratamiento de los tumores colorrectales. Así tenemos el estudio de Lorenz y $\mathrm{col}^{28}$ en el que se emplea una infusión de 5 días $/ 4$ semanas de 5-FU modulado con FC intraarterial. La adherencia al protocolo fue escasa y la toxicidad no fue despreciable la la que probablemente habrá contribuido el leucovorín por vía arterial). Otro estudio que tampoco pudo demostrar diferencias fue el de Rudroff y col${ }^{29}$ (sólo 42 pacientes) en el que se empleó también una infusión de 5 -FU de 5 días/4 semanas junto con mitomicina $C$ intrarterial. En este caso las diferencias que existen a favor del tratamiento intraarterial no pudieron ser significativas a pesar del mejor control local, a causa del pequeño número de la muestra (Tabla III).

Otra cuestión sin resolver es el papel que pueden desempeñar las combinaciones de estos fármacos nuevos con fluoropirimidinas (Folfox, Folfiri, Saltz...). Desgraciadamente esta es una cuestión por resolver incluso en el ámbito de los estadios III del cáncer de colon. Lo que sí parece claro es que es factible la administración de el 5-FU intraarterial junto con las nuevas drogas por vía $\mathrm{IV}^{30}$, e incluso junto a las nuevas drogas por vía arterial ${ }^{31}$.

La experiencia acumulada en la literatura sobre diversos estudios fase I, II, y III es muy amplia por lo que la aplicación de dicha modalidad podría ser implantada al ámbito español sin dificultades insalvables ${ }^{32-39}$, aunque sin despreciar la 
TABLA III

Estudios aleatorizados con quimioterapia intraarterial complementaria

\begin{tabular}{|c|c|c|c|c|c|c|}
\hline \multirow[b]{2}{*}{ Autor } & \multirow[b]{2}{*}{ Tto } & \multirow{2}{*}{$\begin{array}{l}\text { № } \\
\text { Enf }\end{array}$} & \multicolumn{2}{|c|}{ Supervivencia } & \multicolumn{2}{|c|}{ Recurrencias } \\
\hline & & & $\%$ & Mediana & Cualquiera & Hepática \\
\hline \multirow[t]{2}{*}{ Kemeny MM (25) } & ${ }^{*}$ Control & 56 & 52.7 & $47.5 \mathrm{~m}$ & $77.8 \%$ & $68 \%$ \\
\hline & ${ }^{*}$ FUDR IA + Fu IV & 53 & 61.5 a 4 años & $34.2 \mathrm{~m}$ & $53 \%$ & $50 \%$ \\
\hline \multirow[t]{2}{*}{ Kemeny N (24) } & ${ }^{*} \mathrm{Fu}+\mathrm{FC}$ & 82 & 72 & $59 \mathrm{~m}$ & 59 & $41 \%$ \\
\hline & ${ }^{*} \mathrm{Fu}+\mathrm{FC}$ IV + FUDR IA & 74 & 86 a 2 años & $72 \mathrm{~m}$ & 45 & $11 \%$ \\
\hline \multirow[t]{2}{*}{ Lygidakis NJ (27) } & ${ }^{*}$ Control & 20 & & $11 \mathrm{~m}_{-}$ & & \\
\hline & *quimio-inmunoterapia IA & 20 & & $30 \mathrm{~m}$ & & \\
\hline \multirow[t]{2}{*}{ Lorenz M (28) } & ${ }^{*}$ Control & 113 & & $40.8 \mathrm{~m}$ & & $36.7 \%$ \\
\hline & $* 5 \mathrm{Fu}+\mathrm{FC}$ IA & 113 & & $34.5 \mathrm{~m}$ & & $33.3 \%$ \\
\hline \multirow[t]{2}{*}{ Tono T (26) } & ${ }^{*}$ Control & 10 & 50 & $33 m_{-}$ & 80 & $60 \%$ \\
\hline & ${ }^{*} 5 F_{u}$ IA & 9 & 78 a 5 años & $72+m$ & 33 & $11 \%$ \\
\hline \multirow[t]{2}{*}{ Rudroff C y col (29) } & ${ }^{*}$ Control & 16 & 25 & & & \\
\hline & ${ }^{*} 5 \mathrm{Fu}+\mathrm{MMC}$ IA & 14 & 31 a 5 años & & & \\
\hline
\end{tabular}

*sup a 4 años; m, meses; Enf, enfermos; _, estadísticamente significativo

TABLA IV

Estudios abiertos de quimioterapia complementaria tras la resección de metástasis hepáticas

\begin{tabular}{lllll}
\hline Estudio & Fase & No enf & QT IA & QT IV \\
\hline EORTC-40983 * & III & 330 & - & Oxaliplatino/5Fu/FC \\
MSKCC-98072 & I-I & 50 & Floxuridine dexametasona & CPT1 1 \\
NCCTG-N9945, & II & $15-75$ & floxuridina dexametasona & oxaliplatino capecitabina \\
ACOSOG-Z05032 & Phase II & $28-94$ & floxuridina & Oxinotecan \\
MDA-ID-02636 * & Phase II & 80 & - & Oxaliplatino \\
MSKCC-03005 & Phase I & $2-48$ & floxuridina dexametasona & Oxaliplatino \\
& & & &
\end{tabular}

* con quimioterapia neoadyuvante y adyuvante; 5FU, 5-Fluorouracili; VO, Vía oral; QT, quimioterapia; IV, intravenoso; IA, intraarterial.

potencial toxicidad (sobre todo del Fudr) que podría impactar negativamente en la supervivencia ${ }^{40}$.

En este momento, en el ámbito de la oncología médica en España es habitual administrar tratamiento complementario con esquemas tipo Folfox o Folfiri tras la resección de metástasis hepáticas. Realmente la evidencia de esta indicación está basada fundamentalmente en la lógica y no en el empirismo, por lo que hasta que no tengamos resultados de los estudios que actualmente están en marcha, tanto con quimioterapia IA como con quimioterapia IV, el mayor peso de la evidencia recae sobre los tratamientos IA con fluoropirimidinas. En la Tabla IV se resumen los estudios abiertos registrados en esta patología en la página del "National Cancer Institute" http://cancer.gov/cancerinformation en el mes de Febrero de 2004. El estudio de la EORTC 40983 aclarará el papel de la quimioterapia IV; más adelante tendrá que aclararse cual es la vía idónea de utilización de fluoropirimidinas (IA vs IV) con posteriores estudios aleatorizados.

\section{Técnicas para la realización de quimioterapia intraarterial}

Existen dos formas de realizarla. Una de ellas es la cateterización de la arteria hepática de forma percutánea, lo cual requiere un procedimiento invasivo previo a cada uno de los tratamientos. Tras la punción de la arteria femoral, de forma retrógrada se alcanza primero el tronco celiaco y después se llega a la arteria hepática. Antes de proceder a la administración de la medicación hay que sobrepasar la salida de las arterias pilóricas y gastroduodenal (ramas de la arteria hepática). Esta estrategia de tratamiento es poco utilizada por engorrosa.

La otra manera de realizar estos tratamientos es mediante la implantación durante el acto quirúrgico (habitualmente en la resección de las metástasis hepáticas) de un catéter en la arteria gastroduodenal, en la entrada en la arteria hepática común. El extremo proximal del catéter se puede conectar a 
un reservorio subcutáneo (análogo a los que todos conocemos para la administración de quimioterapia IV) o a una bomba de infusión subcutánea programable que se rellena a través de una membrana de silicona. En el primer caso la administración se realiza a través de infusores "externos" y el reservorio subcutáneo queda alojado sobre las últimas costillas flotantes.

En cualquiera de los dos casos hay que proceder durante el acto quirúrgico a la liberación de la arteria gastroduodenal, desde su salida de la arteria hepática común hasta su introducción en la cabeza del páncreas (usualmente un recorrido de 2-4 cm), a la ligadura y sección de las ramitas colaterales de la arteria hepática común y propia, incluyendo la arteria pilórica, que pueden irrigar al antro gástrico y duodeno y a realizar una colecistectomia.

\section{Bibliografía}

1. Gilbert HA, Kagan AR. Metastases: Incidence, detection, and evaluation without histologic confirmation. In: Weiss L, ed. Fundamental aspect of metastasis. Amsterdam: North-Holland Publishing, 1976; 385-405.

2. Taylor I, Machin D, Mullee M, Trotter G, Cooke T, West C. A randomized controlled trial of adjuvant portal vein cytotoxic perfusion in colorectal cancer. Br J Surg 1985; 72: 359-63.

3. Gray BN, de Zwart J, Fisher R, y col. The Australia and New Zealand trial of adjuvant chemotherapy in colon cancer. In Salmon SE, ed. Adjuvant therapy of cancer, ed 5. New York: Grune \& Stratton.

4. Wereldsma CJC, Bruggink ERM, Meijer WS, Roukema JA, Van Putten WD. Adjuvant portal liver infusionin colorectal cancer with 5 -fluorouracil/heparin versus urokinase versus control. Results of a prospective randomized clinical trial (colorectal adenocarcinoma trial). Cancer 1990; 65:425-32.

5. Swiss Group for Clinical Cancer Research (SAKK). Long Term results of single course of adjuvant intraportal chemotherapy for colorectal cancer. Swiss Group for Clinical Cancer Research (SAKK). Lancet 1995; 345:349-53.

6. Beart RW, Moertel CG, Wieand HS y col. Adjuvant therapy for respectable colorectal carcinoma with fluorouracilbadministered by portal vein infusion. Arch Surg 1990; 125: 897-901.

7. Wolmark N, Rockette H, Wickerham DL, y col. Adjuvant therapy of Duke's $A, B$, and $C$ adenocarcinoma of the colon with portal vein infusion: preliminary results of National Surgical Adjuvant Breast and Bowel Project Protocol C-02. J Clin Oncol 1990; 8:1466-75.

8. Fielding LPH, Grace RH. Randomized controlled trial of adjuvant chemotherapy by portal vein perfusion after curative resection for colorectal adenocarcinoma. Lancet 1992; 340:502-6.

9. Liver infusion Meta-analysis group. Portal vein Chemotherapy for colorectal cancer: a meta-analysis Group. J Natl Cancer Inst 1997; 89:497-505.

10. Reapraisal of hepatic arterial infusion in the treatment of non resectable liver metastases from colorectal cancer. Meta-analysis Group in Cancer. J Natl Cancer Inst 1996; 88: 252-8.

11. Rougier $P$, Laplanche $A$, Huguier $M, y$ col Hepatic arterial infusion of floxuridine in patients with liver metastases from colorectal carcinoma: long-term results of a prospective randomised trial. J Clin Oncol 1992; 10:1986-8.
12. Kemeny N, Daly J, Reichman B, Séller N, Botet J, Oderman P. Intrahepatic or systemic infusion of fluorodeoxyuridine in patients with liver metastases from colorectal carcinoma. Ann Intern Med 1987; 107:459-65.

13. Hohn $D$, Stagg $R$, Friedman $M$, y col. A randomised trial of continuous intravenous versus hepatic intraarterial floxuridine in patients with colorectal cancer metastatic to the liver: the northern California oncology group trial. J Clin Oncol 1989; 7:1646-54.

14. Chang AE, Schneider PD, Sugarbaker PH. A prospective randomised trial of regional versus systemic continuous 5-fluorodeoxyuridine chemotherapy in the treatment of colorectal liver metastases. Ann Srurg 1987; 206:685-93.

15. Martin JK jr, O'Connell MJ, Wieand HS, y col. Intraarterial floxuridine versus systemic fluorouracil for hepatic metastases from colorectal cancer: A randomized trial. Arch Surg 1990; 125:1022-7.

16. Rougier PH, Hay JM, Olivier JM, y col. A controlled multicentric trial of intrahepatic chemotherapy vs standard palliative treatment for colorectal liver matastases. Resúmenes de ASCO 1990; 9:403.

17. Expectancy or primary chemotherapy in patients with advanced asymptomatic colorectal cancer: a randomised trial. Nordic Gastrointestinal Tumor Adjuvant Therapy Group.-J Clin Oncol 1992; 10:904-11.

18. Curley SA, Roh MS, Chase JL. Adjuvant hepatic arterial infusion chemotherapy after curative resection of colorectal liver metastases. Am J Surg 1993; 66:743-6.

19. Lorenz M, Staib-Sebler E, Loch B. The value of postoperative hepatic arterial infusion following curative liver resection. Anticancer Res 1997; 17:3825-33.

20. Okuno K, Shigeoka H, Lee YS. Adjuvant hepatic arterial IL-2 and MMC, 5-FU after curative resection of colorectal liver metastases. Hepato-gastroenterology 1996; 43:688-91.

21. Ambiru S, Miyazaki $\mathrm{H}$, Ito $\mathrm{H}$. Adjuvant regional chemotherapy after hepatic resection for colorectal metastases. Br J Surg 1999; 86:1025-31

22. Kokudo N, Seki M, Ohta $H$, et al. Effects of systemic and regional chemotherapy after hepatic resection for colorectal metastases. Ann Surg Oncol 1998; 5:706-12.

23. Nonami T, Takeuichi Y, Yasui M. Adjuvant chemotherapy after partial hepatectomy for metastatic colorectal carcinoma. Semin Oncol 1997; 24:s6-130.

24. Kemeny $N$, Huang $Y$, Cohen AM, y col. Hepatic arterial infusión of chemotherapy after resection of hepatic metastases from colorectal cancer. N Engl J Med 1999; 34:2039-48.

25. Kemeny M, Adak S, Gray B, y col. Combined-modality treatment for respectable metastatic colorectal carcinoma to the liver: surgical resection of hepatic metastases in combination with continuous infusion of chemotherapy- An intergroup Study. J Clin Oncol 2002; 20:1499-505.

26. Tono $T$, Hasuike $Y$, Ohzato H, Takatsuka $Y$, Kikkawa N. Limited but definite efficacy of prophylactic hepatic arterial infusion chemotherapy after curative resection of colorectal liver metastases: A randomized study. Cancer 2000; 88:1549-56.

27. Lygidakis $\mathrm{Ni}$, Ziras $\mathrm{N}$, Parissis J. Resection versus resection combined with adjuvant pre- and post-operative chemotherapyinmunotherapy for metastatic colorectal cancer. A new look at an old problem. Hepatogastroenterology 1995; 42:155-61.

28. Lorenz M, Muller HH, Schramm H, y col. Randomized trial of surgery versus surgery followed by adjuvant hepatic arterial infusion with 5-fluorouracil and folinic acid for liver metastases of 
colorectal cancer. German Cooperative on Liver Metastases. Ann Surg 1998; 228:756-62.

29. Rudroff C, Altendorf-Hoffmann A, Stangl R, Scheele J. Prospective randomised trial on adjuvant hepatic-artery infusion chemotherapy after RO resection of colorectal liver metastases. Arch Surg 1999; 384:243-9.

30. Kemeny N, Gonen M, Sullivan D, y col. Phase I study of hepatic arterial infusion of floxuridine and dexamethasone with systemic irinotecan for unresectable hepatic metastases from colorectal cancer. J Clin Oncol 2001; 15;19:2687-95.

31. Kern W, Beckert B, Lang N, y col. Phase I and pharmacokinetic study of hepatic arterial infusion with oxaliplatin in combination with folinic acid and 5-fluorouracil in patients with hepatic metastases from colorectal cancer. Ann Oncol 2001; 12:599-603.

32. Porta $C$, Danova $M$, Accurso $S$. Sequential intrahepatic and systemic fluoropyrimidine-based chemotherapy for metastatic colorectal cancer confined to the liver. A phase II study. Cancer Chemother Pharmacol 2001; 47:423-8.

33. Berlin J, Merrick HW, Smith TJ, Lerner H. Phase II evaluation of treatment of complete resection of hepatic metastases from colorectal cancer and adjuvant hepatic arterial infusion of floxuridine: an Eastern Cooperative Oncology Group Study (PB083). Am J Clin Oncol 1999; 22:291-3.

34. O'Connell MJ, Nagorney DM, Bernath AM, y col. Sequential intrahepatic fluorodeoxyuridine and systemic fluorouracil plus leucovorin for the treatment of metastatic colorectal cancer confined to the liver. J Clin Oncol 1998; 16: 2528-33.

35. Lorenz M, Mueller HH, Mattes E, y col. Phase II study of weekly 24-hour intra-arterial high-dose infusion of 5-fluorouracil and folinic acid for liver metastases from colorectal carcinomas. Ann Oncol 2001; 12:321-5.

36. Copur MS, Capadano M, Lynch J, y col. Alternating hepatic arterial infusion and systemic chemotherapy for liver metastases from colorectal cancer: a phase II trial using intermittent percutaneous hepatic arterial access. J Clin Oncol 2001; 19:2404-12.

37. Van Riel JM, van Groeningen CJ, Albers SH, y col. Hepatic arterial 5-fluorouracil in patients with liver metastases of colorectal cancer: single-centre experience in 145 patients. Ann Oncol 2000; 11:1563-70.

38. Kurihara A, Nakazaki $H$, Watanabe $M$, y col. Hepatectomy and intraarterial infusion chemotherapy for liver metastasis from colorectal cancer. Gan To Kagaku Ryoho 2002; 29:2104-7.

39. Hidalgo OF, Rebollo J, Viéitez JM. Itraarterial hepatic treatment with carboplatin (CBDCA) and 5-Fluorouracil (5-Fu) in metastases from colorectal carcinoma. Am J Clin Oncol; 16:30-3.

40. Berlin J, Merrick HW, Smith TJ, Lerner H. Phase II evaluation of treatment of complete resection of hepatic metastases from colorectal cancer and adjuvant hepatic arterial infusion of floxuridine: an Eastern Cooperative Oncology Group Study (PB083). Am J Clin Oncol 1999 Jun 22; 291-3. 